\title{
El recurso humano, factor de competitividad en el sector salud
}

\section{The human resource factor of competitiveness in the health sector}

\author{
Remedios Piteres Redondoํㅜ, Marcos Cabarcas Velázques², Hugo Gaspar Hernández ${ }^{3}$
}

\section{Resumen}

Objetivo: realizar un análisis del papel que juega la gestión del recurso humano al interior de una organización que busca la competitividad.

Resultados y conclusiones: el talento humano de una empresa constituye el activo más valioso de la misma, es por esto que una buena gestión del talento humano se debe caracterizar por potenciar el capital humano en beneficio de los objetivos, visualizando a los empleados como socios para que a través de sus talentos desarrollen las estrategias, antes que a través de acciones administrativas generando una ventaja competitiva en el mercado e impactando positivamente los diversos programas y políticas, así como los estados financieros de la empresa.

\section{Palabras Claves:}

Recurso humano, ompetitividad, servicio de salud, sostenibilidad.

\begin{abstract}
Objective: to carry out an analysis of the role that human resource management plays within an organization that seeks competitiveness.

Results and conclusions: the human talent of a company is the most valuable asset of the same, which is why a good human talent management should be characterized by enhancing human capital for the benefit of the objectives, visualizing employees as partners for that through their talents develop strategies, rather than through administrative actions generating a competitive advantage in the market and positively impacting the various programs and policies, as well as the financial statements of the company.
\end{abstract}

1 Universidad de la Guajira, Correo electronico: zhurayacosta@hotmail.com

2Universidad del Atlántico y del SENA Correo electronico: zhurayacosta@hotmail.com

3 Autor de correspondencia, Universidad del Atlántico, Correo electronico: zhurayacosta@hotmail.com 


\section{Introducción}

Una buena gestión del talento humano se caracteriza por potenciar el capital humano en beneficio de los objetivos organizacionales, y visualizar a los empleados como socios que desarrollan las estrategias de la empresa por medio de sus talentos, antes que a través de acciones administrativas [1]. Mediante esta visión, se logra compromiso, empoderamiento e identidad corporativa en las personas, lo que cambia la antigua tendencia sobre el personal.

De acuerdo con varias investigaciones recientes, el recurso humano es uno de los pilares fundamentales de la empresa, pues, se reconoce como un patrimonio intangible [2], a través del cual se puede implementar, reacomodar, adaptar y hacer factible cualquier adelanto tecnológico, concepto teórico o aplicación práctica, lo que le otorga mayor vitalidad a las organizaciones

Las teorías relacionadas afirman que sin gente eficiente es imposible que una organización logre sus objetivos [3]. Por eso, en la actualidad, la gestión del talento humano se ha convertido en un enfoque estratégico de dirección, cuyo objetivo se centra en obtener la máxima creación de valor para la organización a través de un conjunto de acciones dirigidas a disponer en todo momento de los conocimientos, capacidades y habilidades de los miembros de la organización, con miras a la obtención de resultados competitivos en el entorno actual y futuro [4]. Así mismo, los objetivos organizacionales de las empresas que pretenden ser exitosas y llegar a la máxima rentabilidad se basan en la operación eficiente, en tiempos óptimos, con costos moderados y que no ponga en riesgo el ambiente interno ni conduzca al conflicto. 


\section{Componentes de la gestion humana empresaral}

Los preceptos vanguardistas han incorporado tres componentes importantes a la gestión del talento humano: los objetivos organizacionales, que se constituyen en las metas trazadas por la empresa; la visión de la organización como una estructura, con todos sus componentes y cultura; y el valor de las personas, como el capital intelectual de la organización [5]. En este sentido, la estrategia radica en la capacidad de mantener la ventaja competitiva de las empresas a partir del valor que el talento humano agrega a la organización.

En esta misma línea, se propone equilibrar las capacidades de la empresa con las demandas del entorno y, por ende, al capital humano de la organización para afrontar el desafío. Además, se da por sentado que los gerentes de talento humano se enfrentan a tres desafíos estratégicos básicos: primero, el apoyo a los esfuerzos por mejorar la productividad y el desempeño corporativos; segundo, proporcionar a los empleados un papel más amplio en los esfuerzos por mejorar el desempeño; y tercero, la participación de los recursos humanos en el diseño (y no solo en la ejecución) del plan estratégico de la empresa [6]. En otras palabras, la estrategia está centrada en las personas, y su éxito está determinado por el nivel de participación y compromiso del talento humano y su nivel de eficacia.

Teniendo en cuenta que las empresas de salud tienen una dinámica diferente a otros sectores económicos del país, la disposición de los mejores recursos humanos puede garantizar en ellas la calidad de las actividades desarrolladas, en especial, porque en este sector el objetivo es curar y mejorar la salud de la gente, lo que la diferencia de todos los otros negocios [7]. Varios estudios indican que la gestión eficaz del talento humano tiene una directa relación con sus utilidades [8]. Por lo tanto, generar estrategias para garantizar el bienestar integral del talento humano en el sector de la salud constituye un factor diferencial y decisorio para mantener este importante recurso en un alto nivel de competencia, a fin de que ofrezca un servicio de calidad y una atención humanizada. La articulación de los procesos con las políticas y el marco estratégico, iniciando desde el proceso de selección hasta un adecuado proceso de desvinculación, incide en la mejora continua del servicio [9]. De acuerdo con esto, la principal complejidad en el sector salud radica en dos factores: el encuentro de personal idóneo para cada una de las funciones y especialidades (que son muchas) y el reconocimiento de la remuneración correspondiente [10], la cual depende de muchas más variables que el común de las empresas. 


\section{Talento humano en el sector de la salud}

En su mayoría, las empresas dedicadas a la salud presentan particularidades como: funcionamiento por turnos durante las 24 horas del día, lo cual en ocasiones genera agotamiento físico y mental en el equipo humano [11]; el hecho de que la mayoría de los profesionales especializados se rotan de empresa en empresa, generando retrasos en la prestación del servicio y el contar con grupos de colaboradores de diferentes especialidades, a lo que se añade la necesidad de cubrir perfiles muy variados, que van desde seguridad y limpieza hasta cirujanos de alta complejidad como, por ejemplo, expertos en trasplantes. Debido a esto último, en la mayoría de casos no se cuenta con el personal suficiente para cubrir las necesidades.

Por sus particularidades, la administración del talento humano en el sector salud es un tema que cobra cada vez más fuerza y protagonismo en el desempeño de los sistemas de salud en términos de universalidad, calidad, eficiencia y equidad [12].

Ahora bien, en la actualidad, Colombia atraviesa por una crisis en el sector de la salud que ha desencadenado, entre muchas cosas, una inestabilidad laboral y financiera en los profesionales en salud. Diversas organizaciones del sector han denunciado, por ejemplo, que además de que les pagan con varios meses de retraso, las tarifas de pago de las entidades que los contratan, sean EPS o IPS, se fijan de manera arbitraria [13]. Durante años, en efecto, estas tarifas no han sido actualizadas, lo que va en contra de la calidad de vida y poder adquisitivo de los profesionales, que invierten en promedio 10 años para alcanzar cierto nivel de especialidad.

Pese a la importancia de contar con un talento humano competente para cumplir sus servicios misionales, la salud en el país se ha convertido en un negocio donde solo algunos obtienen beneficios [14]. También a nivel mundial, se han venido desarrollando estrategias de análisis para el manejo del personal médico, dado que en general el talento humano del sector salud presenta aspectos a mejorar. Y es que la problemática del sector abarca desde el análisis de las necesidades territoriales de profesionales para atender a los problemas de salud de la población hasta el control y la regularización de tarifas, con el fin de establecer unos honorarios e incentivos que garanticen la adecuada prestación de los servicios de salud y eviten la migración masiva de profesionales.

Más en concreto, de acuerdo con el Ministerio de Salud, las estadísticas del país referidas a la oferta de servicios en el sector expresan que la formación de competencias y perfiles de estos recursos humanos son insuficientes para potenciar mayor capacidad resolutiva [15], lo que profundiza la baja respuesta institucional y genera el represamiento de las demandas. Esto conlleva a que, en algunos momentos, durante la prestación del servicio, los profesionales de la salud se expongan a agresiones verbales o físicas de los usuarios e incrementen así sus niveles de riesgo psicosocial.

Por otro lado, la oferta de servicio en relación a los prestadores públicos indica una disminución de 
un $2.8 \%$ en los primeros niveles de atención entre los años 2013 y 2016. Adicionalmente, los niveles de atención básicos presentan alta obsolescencia tecnológica, así como deficiencia en cantidad, pertinencia, calidad del personal de salud y de infraestructura, situaciones todas que se evidencian en la oferta pública, donde la inversión en reposición ha sido escasa [16].

Reconociendo la importancia del recurso humano, el Ministerio de Salud elaboró un documento llamado "Estrategia de incentivos al talento humano en salud tarea de todos" [17], que, partiendo de la motivación del talento humano como pilar estratégico de una organización, busca comprender la problemática y desarrollar estrategias encaminadas a crear un plan de incentivos acorde con las necesidades de la organización, que puedan disminuir costos y aumentar la productividad de la misma. Según el documento, el objetivo de la investigación consistió en conocer los incentivos no financieros que pueden disminuir los costos y a la vez aumentar la productividad.

Temiendo en cuenta que la crisis que enfrenta el talento humano en salud es global, conviene fortalecer los resultados de su desempeño para el logro de los llamados "objetivos del milenio" [18]. De acuerdo con la Alianza Mundial para los Recursos Humanos de salud 2008, en general, se aprecia en el sector: bajos salarios, condiciones de trabajo deficientes, obstáculos geográficos, sistemas de salud "abandonados" e insuficientemente dotados de recursos, así como prácticas y estructuras deficientes de planificación y gestión de los recursos humanos [19]. Según World Health Organization, entre los puntos identificados como los más críticos en materia de condiciones laborales aparecen:

- Pesadas cargas de trabajo

- Falta de autonomía

- Supervisión y apoyo deficientes

- Prolongados horarios de trabajo

- Lugares de trabajo inseguros

- Estructuras de carrera profesional inadecuadas

- Escasa remuneración

- Suministros, instrumentos e información insuficientes

Según la Organización Mundial de la Salud (OMS), para combatir los problemas del sector en lo concerniente a talento humano se debe contar con trabajadores adecuados, competencias adecuadas, el lugar adecuado, y hacer las cosas adecuadas [20]. La OMS afirma que solo así los trabajadores de la salud contarán con la capacidad para responder a las crisis, enfrentar las brechas actuales y anticiparse al futuro.

La prestación de servicios de salud es una actividad compleja que requiere un alto nivel de responsabilidad. Para ello se precisa un conjunto de capacidades que van desde las interpersonales hasta las muy técnicas y especializadas [17]. Pero es claro que los profesionales de salud son el recurso más importante del sector y que los recursos humanos en este campo comprenden entre el $40 \%$ y el $90 \%$ de los presupuestos de los servicios de salud [18]. Con todo, por mucho tiempo los sistemas de salud tendieron a considerar el recurso humano como un costo y no como una inversión. La cuestión ha cambiado totalmente en los estudios de hoy, como lo indica el concepto de incentivos eficaces, que se entienden como una motivación para los empleados que los lleva a disfrutar las tareas y a comprometerse con la organización.

Como citar este artículo: R. Piteres, M. Cabarcas., y H. Gaspar, "El recurso humano factor de competitividad en el sector salud", Investigación e Innovación en Ingenierias, vol. 6, n. 1, pp. 93 101., 2018 DOI: https://10.17081/invinno.6.1.2778 
Los incentivos constituyen, en efecto, herramientas que las organizaciones pueden emplear para atraer, retener, motivar, dar satisfacción a su personal y mejorar sus resultados. Por esta razón son empleados por la mayoría de las organizaciones, sin embargo, debe entenderse que los incentivos no son suficientes por sí solos. Por eso, el gran reto para Colombia y el resto de países consiste en obtener una combinación equilibrada entre lo financiero y lo no financiero, a fin de obtener resultados perdurables. De lo contrario, solo se constituirán en paliativos con resultados cortoplacistas y sin trascendencia para la institución y para el sistema. En un marco conceptual global, se reconocen los siguientes factores como influenciadores en la motivación personal de los trabajadores de salud [19 - 23]:

- Factores determinantes personales: Lo conforman las necesidades personales; el concepto de sí mismo; las expectativas de resultados o las consecuencias de las actividades laborales.

- Contexto de la organización: Que se expresa en salarios; ventajas; sistemas claros y eficientes;

- sistemas de gestión de los recursos humanos; observaciones acerca de los resultados; cultura de la organización.

- Contexto social y cultural: Comprendido en las expectativas y observaciones de la comunidad.

- Reforma del sector de salud: Que busque comunicación y dirección, y coherencia con los valores personales de los trabajadores. 


\section{Conclusión}

Las reflexiones más perceptibles de este trabajo se enfocan en revisar la normatividad en materia del talento humano del sector salud, pues esta debe enmarcarse en las diferentes políticas que promuevan el bienestar de la población, como marco de referencia para adaptar las políticas e integrar a los profesionales de salud. Solo así se podrá estructurar una mejora sostenible en el gremio y establecer buenas prácticas de bienestar, es decir, dirigidas a mejorar el clima laboral, donde el trabajador se desempeña la mayor parte de su vida. Esto afectará de manera positiva la prestación de los servicios de salud, brindando al usuario o paciente final calidez, amabilidad, seguridad y tranquilidad en su proceso de recuperación de la salud. 


\section{Referentes}

1. G. Calderón, Aproximación a un modelo de gestión humana que agregue valor a la empresa colombiana. Bogotá: Universidad Nacional de Colombia, 2008.

2. G. Calderón, y C. M. Álvarez, "Características y sentido de las prácticas de gestión humana en las pequeñas empresas". Revista Universidad Eafit, vol. 42, n. 142, pp. 26-45, 2006.

3. Calderón, Naranjo y Álvarez. “Gestión humana en las organizaciones un fenómeno complejo: evolución, retos, tendencias y perspectivas de investigación”. Cuadernos de Administración, vol. 19, n. 32, pp. 225-54, 2006.

4. L. Merina, \& F. González "La gestión por competencias y su impacto sobre el compromiso organizacional", 2010. Disponible en: http://copsa.cop.es/congresoiberoa/base/trabajo/orgr36. htm.

5. N. Bordoni, "Formación de recursos humanos en salud para el desarrollo", Medicina y sociedad, 2014. Disponible en http:/ / www.medicina y sociedad.org.ar/.

6. C. Borrell y W. Malmusi, "Investigación y determinantes sociales de salud e inequidades de salud: evidencia en todas las políticas”. Gac. Sanit., vol. 24 (Supp1), pp. 101-108, 2010.

7. J. Breilh, "La epidemiología crítica: una nueva forma de mirar la salud en el espacio urbano". Salud colectiva, vol. 6, n. 1, pp. 1-19, 2010.

8. J. Bruner, "La educación puerta de la cultura". Madrid: Visor. Buyayisqui, M.P.; Bordoni, N.; Garbossa, G. (2013). “Overcoming Language and Cultural Barriers. A Graphical Communication Tool to Perform a Parasitological Screening in two Vulnerable Populations from Argentina". Journal of Health Communication, vol. 18, n. 1, pp. 92-104, 2013.

9. M. Cadile, M. Aulicino, I. Duré y N. Bordoni, "Vinculación entre la Universidad y el Sistema de Salud. Un modelo en desarrollo", Revista Maestría Salud Pública UBA, vol. 6, n. 12, 2006.

10. R. Saltman, et al. "Governing Public Hospitals. Reform strategies and the movement towards institutional autonomy. WHO". European Observatory on Health Systems and Policies. Observatory Studies Series, vol. 25, 2011.

11. H. Orwa Bula. "Evolution and Theories of Entrepreneurship: A Critical Review on the Kenyan Perspective". International Journal of Business and Commerce, vol. 1, n. 11, pp. 81-96, 2012.

12. N. Velasco, "La salud de la mano de la ingeniería industrial". Revista Contacto universidad de los Andes, 2013. Disponible en: https://revistacontacto.uniandes.edu.co/index.php/la-salud-dela-mano-de-la-ingenieria-industrial.

13. DA. Rodríguez, LMJ. Vidal, RA. Cuellar, GBD. Martínez y AYM. Cabrera, "Desarrollo de la informatización en Hospitales". INFODIR, vol. 21, pp. 3-15, 2015.

14. G. Arenas, Los riesgos del trabajo y la salud ocupacional en Colombia. Bogotá D.C.: Editorial Legis, 2014.

15. M.P. Alfonso, "Empresas y Derechos Humanos: Una Responsabilidad en Construcción". Facultad de Derecho y Ciencias Políticas. Vol. 43, nº. 118, pp. 349-385, 2011.

16. Ministerio de Salud (MINSALUD). "Incentivos al Talento Humano en Salud". "Estrategia 
de incentivos al talento humano en salud "tarea de todos" (2016). Disponible en: https://www. minsalud.gov.co/sites/rid/Lists/BibliotecaDigital/RIDE/VS/TH/1.estrategia-tarea-todosincentivos-minsalud.pdf.

17. Departamento para La Prosperidad Social. "Plan Estratégico. Bogotá, 2015". Obtenido de: htpp:/ /apps.dps.gov.co/documentos/11062_Plan_estrat\%C3\%A9gico_sectorial_2015.pdf

18. H. Hernández, "La gestión empresarial, un enfoque del siglo $X X$, desde las teorías administrativas científica, funcional, burocrática y de relaciones humanas", Revista Escenarios. Vol. 9, No. 1, pp. 38-51, 2011.

19. J. Ortega, H. Hernández, H. Mariano y A. Herrera, “Calidad Estratégica en los Servicios de Salud", Centro de investigación y proyectos (CINPRO) Corporación Universitaria Latinoamericana, 2015.

20. C. Merino, "Contexto emprendedor en el sector de la salud", Cuadernos Latinoamericanos de Administración, vol. 6, n. 11, pp. 45-58, 2010.

21. L. Ferrer Castellanos, K. González Insignares and L. Mendoza Vega, “La innovación como factor clave para mejorar la competitividad de las pymes en el departamento del Atlántico, Colombia", Dictamen Libre, no. 16, pp. 21-36, 2015.

22. H. Hernández Palma, «Sistemas de gestión integrados en el sector salud para la optimización de la calidad en el departamento del atlántico,» Dictamen Libre, n² 20, pp. 99-106, 2017.

23. M. Parra y S. Durán, «Desarrollo organizacional y estrategias de tecnoformación en instituciones de educación superior colombianas,» Dictamen Libre, nº 14/15, pp. 14-23, 2014. 